\title{
Efecto del potasio y la densidad de siembra en la producción de papa Solanum tuberosum Grupo Phureja var. Criolla Guaneña
}

\author{
Effect of potassium and seed density on potato production \\ Solanum tuberosum Group Phureja var. Criolla Guaneña
}

\author{
Amanda Silva Parra ${ }^{*}$, Cesar Albornoz Bucheli², Hernando Criollo Escobar ${ }^{3}$ \\ Recibido para publicación: Febrero 27 de 2017 - Aceptado para publicación: Noviembre 23 de 2017
}

\begin{abstract}
RESUMEN
El potasio es un macronutriente que puede mejorar el crecimiento de los cultivos. Se empleó un diseño en bloques completos al azar con el objetivo de evaluar la influencia de cuatro densidades de siembra (50.000, $41.667,33.333,31.250$ plantas $\left.\mathrm{ha}^{-1}\right)$ y tres niveles de potasio $\left(300,250,200 \mathrm{~kg}\right.$ de $\left.\mathrm{K}_{2} \mathrm{O} \mathrm{ha}^{-1}\right)$, sobre el área foliar $(\mathrm{AF})$, índice de área foliar (IAF), índice de crecimiento de cultivo (ICC) e índice de cosecha (IC) en papa Solanum tuberosum Grupo Phureja var. Criolla Guaneña cultivada en una zona de Botana, Altiplano de Pasto, Nariño, Colombia. Todas las evaluaciones se midieron cada 20 días hasta los 120 días después de la siembra (dds), menos el IC al final del ciclo. Se realizó análisis de varianza, comparación de medias Tukey $(P<0,05)$ y regresión entre las variables evaluadas. Las variables fisiológicas presentaron un ajuste significativo a los modelos polinómico de tercer grado, cuadrático y de Hoerl, con coeficientes de determinación superiores a 0,6 , indicando que con 31.250 plantas ha-1 y $250 \mathrm{~kg} \mathrm{~K}_{2} \mathrm{O} \mathrm{ha}^{-1}$ se lograron incrementos en AF de $6000 \mathrm{~cm}^{2}$ y con $200 \mathrm{~kg} \mathrm{~K}_{2} \mathrm{O} \mathrm{ha}^{-1}$ en IAF e ICC de 2,0 y $40 \mathrm{~g} \mathrm{~m}^{2}$ día $^{-1}$ entre 60 a 80 y 80 a 100 dds, respectivamente; sin embargo, con 33.333 plantas ha ${ }^{-1}$ y $250 \mathrm{~kg} \mathrm{~K}_{2} \mathrm{O}$ ha-1 se incrementó el IC $(0,70)(\mathrm{P}<0,05)$. Aunque las regresiones mostraron tendencia a aumentos del IAF e ICC con las prácticas de manejo de producción realizada por los agricultores (41.667 plantas ha ${ }^{-1}$ y 250 $\mathrm{kg} \mathrm{K}_{2} \mathrm{O} \mathrm{ha}{ }^{-1}$ ) entre 60 a 80 dds, no se recomienda ya que se obtuvo un menor IC $(0,60)$ al final del ciclo.
\end{abstract}

Palabras clave: Densidad de plantas, fertilización, luminosidad, nutrientes, tubérculos.

\begin{abstract}
Potassium is a macronutrient that can improve the growth of crops. A randomized complete block design was used to evaluate the influence of four planting densities $(50,000$, $41,667,33,333,31,250$ plants ha-1) and three potassium levels $\left(300,250,200 \mathrm{~kg}\right.$ of $\left.\mathrm{K}_{2} \mathrm{O} \mathrm{ha}^{-1}\right)$ on leaf area (AF), leaf area index (LAI), crop growth index $(\mathrm{CCl})$ and harvest index $(\mathrm{Cl})$ in potato Solanum tuberosum Phureja var. Criolla Guaneña grown in an area of Botana, Altiplano de Pasto, Nariño, Colombia. All evaluations were measured every 20 days until 120 days after sowing (das), except for the IC at the end of the cycle. Analysis of variance, comparison of Tukey means $(\mathrm{P}<0.05)$ and regression among the evaluated variables was performed. The physiological variables presented a significant adjustment to the polynomial models of third degree, quadratic and Hoerl, with coefficients of determination higher than 0.6, indicating that with 31,250 plants ha-1 and $250 \mathrm{~kg} \mathrm{~K}_{2} \mathrm{O}$ $\mathrm{ha}^{-1}$ increments were achieved in $\mathrm{AF}$ of $6000 \mathrm{~cm}^{2}$ and with $200 \mathrm{~kg} \mathrm{~K}_{2} \mathrm{O} \mathrm{ha}^{-1}$ in IAF and ICC of 2.0 and $40 \mathrm{~g} \mathrm{~m}^{2}$ day $^{-1}$ between 60 to 80 and 80 to 100 das, respectively; However, with 33,333 plants ha-1 and $250 \mathrm{~kg} \mathrm{~K}_{2} \mathrm{O} \mathrm{ha}^{-1}$ the IC $(0.70)$ was increased $(\mathrm{P}<0.05)$. Although the regressions showed a tendency to increase the LAI and ICC with the production management practices carried out by the farmers $\left(41,667\right.$ plants ha ${ }^{-1}$ and $\left.250 \mathrm{~kg} \mathrm{~K}_{2} \mathrm{O} \mathrm{ha}^{-1}\right)$ between 60 to 80 das, it is not recommended since a lower IC (0.60) was obtained at the end of the cycle.
\end{abstract}

Key words: Plant density, Fertilization, luminosity, nutrients, tubers.

\footnotetext{
1* PhD. Universidad de los Llanos, Villavicencio, Colombia, Sede Barcelona, Km. 12 vía Puerto López, 3148005493, asilvap@unillanos. edu.co

${ }^{2}$ MSc. Universidad de Nariño, San Juan de Pasto, Colombia.

${ }^{3}$ PhD. Universidad de Nariño, San Juan de Pasto, Colombia.
} 


\section{INTRODUCCIÓN}

Unas óptimas densidades de siembra y dosis de potasio (K) son algunos de los factores importantes para la producción de papa Solanum tuberosum, ya que están asociados con la fisiología del cultivo y el mejoramiento de su calidad y productividad (Santos, 2010).

Después del nitrógeno $(\mathrm{N})$, el $\mathrm{K}$ es el nutriente mineral requerido en mayor cantidad por la mayoría de las plantas, pero en el cultivo de papa es la excepción (Becerra et al., 2007). El orden de requerimiento nutricional en papa es $\mathrm{K}, \mathrm{N}$ y fósforo $(\mathrm{P})$, un rendimiento de $56 \mathrm{t} \mathrm{ha}^{-1}$ de papa, extrae alrededor de 300-100 y $500 \mathrm{~kg}$ $\mathrm{ha}^{-1}$ de N-P $\mathrm{O}_{5}$ y K $\mathrm{K}_{2} \mathrm{O}$ (lerna et al., 2011). El requerimiento de $\mathrm{K}$ para el óptimo crecimiento vegetal está en el rango entre 2 a $5 \%$ del peso seco de las partes vegetativas y tubérculos (Becerra et al., 2007). Cada tonelada de papa cosechada extrae alrededor de $4 \mathrm{~kg}$ de $\mathrm{K}_{2} \mathrm{O}$ en los tubérculos (Sifuentes et al., 2013).

El $\mathrm{K}$ es un activador de muchas enzimas esenciales en la fotosíntesis y la respiración, además, activan enzimas reguladoras, particularmente la piruvato quinasa y las fosfofructoquinasas, necesarias para formar almidón y proteínas (Mohr et al., 2012), en papa el K juega un papel importante en el desarrollo y Ilenado de los tubérculos (Nava et al., 2007).

La respuesta fisiológica del cultivo de papa puede verse afectada por la fertilización y las densidades de siembra, los cuales son factores limitantes de la producción sino son bien manejados (Rodríguez et al., 2009).

El aumento en la densidad de siembra en el cultivo de papa, puede favorecer la producción debido a que se mejora la captura de la radiación fotosintéticamente activa (RFA) del cultivo (Aguilar, 2006). Según De La Casa et al., (2008) el área foliar (AF) es de gran importancia por cuanto determina la capacidad de la planta para interceptar la RFA, necesaria para la producción de biomasa y el llenado de tubérculos (Criollo y García, 2009), que asegura los rendimientos económicos del cultivo de la papa.

Por otra parte, el índice de area foliar (IAF) es un estimador de la productividad y es necesario en la definición del consumo de agua del cultivo (De La Casa et al., 2008). Un mayor IAF es indicativo de mayor productividad, aunque el excesivo desarrollo foliar puede significar un retardo en la tuberización y llenado, así como fallas en la partición de los asimilados a los sitios de mayor consumo (Aguilar, 2006).

La presencia de órganos con déficit de asimilados, influye en la distribución de la materia seca originando variaciones en el índice de cultivo (IC), ya que la producción de asimilados en las hojas y su transporte a los sumideros o vertederos que para el caso son los tubérculos, dependen de varios factores de tipo agronómico y ambiental (Aguilar, 2006), con gran interés el manejo de las densidades de siembra.

Sin embargo, en Colombia, las investigaciones en papa criolla Solanum tuberosum Grupo Phureja var. Criolla Guaneña han estado dirigidas a evaluar el efecto de la fertilización potásica y densidades de siembra sobre la producción (Ríos, 2010), y en optimizar el rendimiento de los cultivares de papa de año (tetraploides) (Madroñero et al., 2013), con pocos estudios sobre sus efectos en la fisiología del cultivo (Rojas, 2014).

El objetivo de esta investigación fue evaluar el efecto de la aplicación de tres niveles de fertilización potásica y cuatro densidades de siembra sobre el comportamiento fisiológico del cultivo de papa Solanum tuberosum Grupo Phureja var. Criolla Guaneña, bajo las condiciones agroclimáticas de la zona de Botana, Altiplano de Pasto, departamento de 
Nariño, Sur de Colombia, con la finalidad de contribuir a optimizar su producción reflejado en el índice de cosecha del cultivo (IC).

\section{MATERIALES Y MÉTODOS}

La investigación se realizó en la Granja Experimental de Botana, perteneciente a la Facultad de Ciencias Agrícolas de la Universidad de Nariño, ubicada en el corregimiento de Botana, Altiplano de Pasto, departamento de Nariño, Sur de Colombia, la zona presenta una altura de $2.820 \mathrm{msnm}$ y las coordenadas geográficas son $01^{\circ} 09^{\prime} 12^{\prime \prime}$ Latitud norte y $77^{\circ}$ $18^{\prime} 31^{\prime \prime}$ Longitud oeste. Esta zona se caracteriza por registrar una temperatura promedio de 12,6 ${ }^{\circ} \mathrm{C}$, RFA de 250 a 358 nm de longitud de onda, humedad relativa del $79 \%$ y una precipitación anual de 820 mm/año (IDEAM, 2013), Según Holdridge (1990) pertenece a la zona de vida de bosque húmedo montano bajo (bh - MB). El suelo es un Vitric haplustand.

El material fue obtenido del Programa de Mejoramiento de papa criolla de la UNAL, cuyas características fenotípicas de la semilla del tubérculo de papa Solanum tuberosum Grupo Phureja var. Criolla Guaneña son de forma redondeada de $3 \mathrm{~cm}$ de diámetro con cinco o más yemas. La semilla se sembró en 20 surcos de $10 \mathrm{~m}$ de largo, con distancias de siembra de 1,40 m entre surcos y 0,40 m entre plantas, depositando tres tubérculos por sitio con tamaño uniforme de $3 \mathrm{~cm}$ de diámetro.

Para fines del establecimiento del experimento se seleccionó el lote denominado terrazas No. 2 , el cual se caracteriza por tener una pendiente del 4\%, pH 5,72, materia orgánica 3,32\%, $\mathrm{N}$ total $0,13 \%$, $\mathrm{P}$ disponible $78,3 \mathrm{mg} \mathrm{kg}^{-1}$ y $\mathrm{K}$ disponible $0,16 \mathrm{cmol}_{\mathrm{c}} \mathrm{kg}^{-1}$; que de acuerdo a Unigarro y Carreño (2005), corresponde a un contenido de $\mathrm{K}$ bajo. La preparación del suelo experimental fue realizada con la aplicación inicial de $2 \mathrm{~L} \mathrm{ha}^{-1}$ de glifosato, 30 días después se realizó una arada con arado de cincel y una rastrillada, posterior a estas labores se aplicó $300 \mathrm{~kg} \mathrm{ha}^{-1}$ de cal agrícola.

De acuerdo con la investigación, se probaron cuatro densidades de siembra, considerando distancias de siembra entre plantas y surcos de 0,20 m x 1,00 m (50.000 plantas/ha), 0,20 m x $1,20 \mathrm{~m}$ (41.667 plantas/ha), 0,30 m x 1,00 m (33.333 plantas/ha) y 0,40 m x 0,80 m (31.250 plantas/ha) y tres dosis de fertilización potásica de 300, 250 y 200 kg K 2 O/ha utilizando como fuente comercial cloruro de potasio $\mathrm{KCl}$.

Para el estudio se utilizó un diseño de bloques completos al azar en un arreglo factorial $3 \times 4$ con 4 repeticiones, donde el factor $\mathrm{A}$ correspondió a las tres dosis de fertilización y el factor $B$, a las cuatro distancias de siembra para un total de 12 tratamientos y 48 unidades experimentales constituidas por cinco surcos cada una. El área total fue de $1.960 \mathrm{~m}^{2}$.

Al momento de la siembra se aplicó a las semillas en cada unidad experimental, Carbofuran para el control de Gusano Blanco (Premnotripex vorax) en dosis de $40 \mathrm{~g} \mathrm{~L}^{-1}$ y Chlorpyrifos para el control de Polilla Guatemalteca (Scrobipalpopsis solanivora) en dosis de $2 \mathrm{~g} \mathrm{~L}^{-1}$. La siembra se realizó colocando 1 tubérculo por sitio, teniendo en cuenta la distancia de siembra y los niveles de fertilización potásica.

El cultivo fue manejado de acuerdo con las labores culturales y de manejo de la papa que se realiza en la región. Se llevó a cabo una aplicación de fosfato diamónico (DAP) en dosis de $575 \mathrm{~kg}$ ha. Para el control de arvenses, a los 10 días después de la siembra (dds), se aplicó glifosato a razón de $5 \mathrm{ml} \mathrm{L}^{-1}$; posteriormente a los 30 dds se realizaron deshierbas manuales hasta llegar al aporque. Los controles fitosanitarios se realizaron cada ocho días con Mancozeb y Clorpirifos en dosis de 2 g y $1 \mathrm{ml} \mathrm{L}^{-1}$, respectivamente. Según los tratamientos, se aplicó $\mathrm{K}$ en forma de $\mathrm{K}_{2} \mathrm{O}$, en tres niveles usando como fuente $\mathrm{KCl}$, el $50 \%$ se 
aplicó al momento de la siembra y el otro 50\% en el aporque.

El testigo correspondió a la fertilización y distancia de siembra que utilizan los agricultores de la zona, el cual correspondió a la aplicación de 250 kg K 2 O/ha y distancias de siembra de 0,20 $\mathrm{m} \times 1$ 1,20 $\mathrm{m}$ (41.667 plantas/ha). A los 20, 40, 60, 80 y 120 dds, cada 20 días, se extrajeron de cada bloque muestras de plantas correspondientes a cada tratamiento, para determinar en cada evaluación el área foliar (AF), índice de área foliar (IAF) e índice de crecimiento de cultivo (ICC).

Para la determinación del área foliar (AF) se utilizó el método alométrico indirecto no destructivo, donde se utilizaron correlaciones alométricas entre magnitudes de las hojas y el AF. Se tomaron 50 hojas de cuatro plantas de papa, seleccionadas aleatoriamente de los surcos centrales de cada parcela y se evaluó la longitud máxima desde la base del pecíolo hasta el extremo del folíolo central terminal y el ancho máximo de las hojas en forma perpendicular a la longitud máxima. Estas hojas se escanearon, para obtener el AF con la ayuda de un programa de procesamiento de imagen digital (ImageJ) (Abramoff et al., 2004); con el objeto de establecer el mejor modelo que exprese el área foliar en función de una de las dimensiones tomadas o de su producto se utilizó el software Curve Expert, el cual permitió determinar el AF de cada planta con base en una o dos mediciones (Schneider, 2012).

Para la determinación del índice de área foliar (IAF) se dividió cada dato de AF con el área ocupada por la planta según el tratamiento (Garnier et al., 2004). El IAF se define como el área de la hoja verde de un solo lado por unidad de área de la superficie del suelo en el dosel de hoja ancha.

Para el índice de crecimiento del cultivo (ICC), el cual relaciona el incremento del peso de la planta por área del suelo en el tiempo, se realizaron ocho evaluaciones, cada 20 días, extrayendo dos plantas por tratamiento de los surcos centrales. Se aplicó la siguiente fórmula para calcular el ICC (Criollo y García, 2009).

Donde,

$$
\operatorname{TCC} \frac{1}{\mathrm{As}} * \frac{\mathrm{dW}}{\mathrm{dT}} \quad \frac{1}{\mathrm{As}} * \frac{\mathrm{W}_{2}-\mathrm{W}_{1}}{\mathrm{~T}_{2}-\mathrm{T}_{1}}
$$

$\mathrm{W}=$ Peso Seco

$\mathrm{T}=$ Tiempo

As $=$ Área de Suelo

Se midió el índice de cosecha (IC) 120 dds, es la relación entre el peso seco del tubérculo y el peso seco total de la planta (incluyendo los tubérculos) (Aguilar, 2006).

Para determinar el contenido de materia seca, se tomaron 10 plantas completas de los surcos centrales de cada bloque y de cada unidad experimental. Cada planta se pesó y empacó en bolsas de papel, se etiquetaron, anotando la fecha, bloque y tratamiento. Posteriormente fueron Ilevadas al laboratorio, donde nuevamente se pesaron por separado y se picaron: raíces, tallos, hojas y tubérculos, se anotaron los datos obtenidos y se empacaron en bolsas de papel etiquetados para someter las muestras al horno a $70{ }^{\circ} \mathrm{C}$, durante 72 horas, al final se pesaron nuevamente las muestras y se anotó los resultados para los cálculos correspondientes de materia seca (Criollo y García, 2009).

Las variables de AF, IAF e ICC se analizaron mediante análisis de regresión tomando el tiempo como variable independiente; los modelos se seleccionaron con base en los mejores valores de $\mathrm{R}^{2}$, y basados en el reporte del paquete estadístico CurveExpert versión 1.3. El índice de cosecha del cultivo (IC), evaluado al final del trabajo, se sometió a análisis de varianza y pruebas de comparación de medias de Tukey $(\mathrm{P}<0,05)$, utilizando el paquete estadístico SAS versión 9.1. 
Silva et al. - Fisiología del cultivo de papa

\section{RESULTADOS Y DISCUSIÓN}

En la figura 1, se ilustran los diferentes modelos de regresión encontrados entre las densidades de plantas y los niveles de potasio estudiados para AF.

Las tendencias de las parábolas indican mayor AF con 50.000 plantas/ha y 250, 300 kg K $\mathrm{O}_{2}$ ha; 41.667 plantas/ha y $200 \mathrm{~kg} \mathrm{~K}$ O/ha; 33.333 plantas/ha y 200, $250 \mathrm{~kg} \mathrm{~K}$ O/ha y 31.250 plantas/ha y $200 \mathrm{~kg} \mathrm{~K}$ O/ha entre los 60 y 80 dds, ajustándose a un modelo polinómico de tercer grado, con $\mathrm{R}^{2}$ superiores a 0,6 , indicando un buen ajuste de los modelos propuestos; la tendencia cuadrática se ajusta mejor a 31.250 plantas/ha con 250 y $300 \mathrm{~kg} \mathrm{~K}_{2} \mathrm{O} / \mathrm{ha}$, con un $\mathrm{R}^{2}$ de 0,85 y 0,86 , respectivamente; mientras que con 50.000 plantas/ha y 200 kg K $20 /$ ha, 41.667 plantas/ha y 250, $300 \mathrm{~kg} \mathrm{~K}$ O/ha y 31.250 plantas/ha con $300 \mathrm{~kg} \mathrm{~K}$ O/ha se ajustaron al modelo de Hoerl presentando un $\mathrm{R}^{2}$ por encima de 0,81 (Figura 1).
En la figura 1 se observa una tendencia a aumentar el AF entre los 60 y 80 dds, con 31.250 plantas/ha y $200 \mathrm{~kg} \mathrm{~K}_{2} \mathrm{O} / \mathrm{ha}$ con valores cercanos a los $6000 \mathrm{~cm}^{2}$, coincidiendo con el desarrollo aéreo e inicio del Ilenado de tubérculos (Fernandes et al., 2011). Al presentarse una menor competencia entre plantas, éstas tienen mayor habilidad para la toma de nutrientes del suelo, agua y luz, como expuesto por Criollo y García (2009), a diferencia de lo encontrado por Aguilar (2006). También es posible que la variedad estudiada se adapta bien a las condiciones lumínicas de altura (Santos et al., 2010), y no requiere de gran cantidad de plantas para alcanzar incrementos en el AF, ya que la obtención y adaptación del material seleccionado se realizó en zonas similares a las de las condiciones del Altiplano. Mahmud et al., (2012) en zona templada reportaron resultados contrarios a los encontrados; al revisar igualmente los resultados de Rodríguez et al., (2004), confirman lo obtenido.
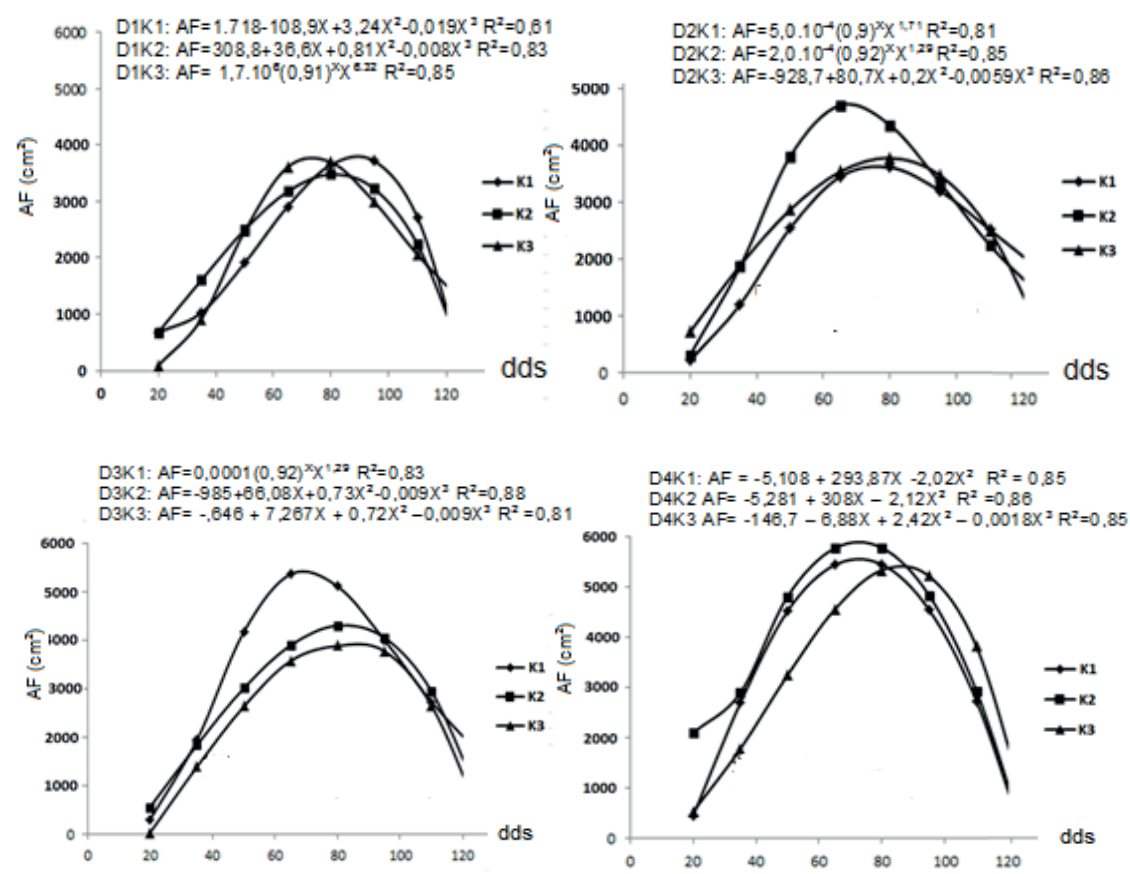

Figura 1. Figura 1. Modelos de regresiones de mejor ajuste para comportamiento del área foliar AF de plantas de papa Solanum tuberosum var. Guaneña, en cuatro densidades de siembra y tres dosis de fertilizante potásico. $\left(\mathrm{D}_{1}=50.000\right.$ plantas $/ \mathrm{ha} ; \mathrm{D}_{2}=41.667$ plantas $/ \mathrm{ha} ; \mathrm{D}_{3}=33.333$ plantas $/ \mathrm{ha} ; \mathrm{D}_{4}=31.250$ plantas $/ \mathrm{ha} ; \mathrm{K}_{1}=300 \mathrm{~kg} \mathrm{~K} \mathrm{O}_{2}$ ha; $\left.\mathrm{K}_{2}=250 \mathrm{~kg} \mathrm{~K} \mathrm{~K}_{2} \mathrm{O} / \mathrm{ha} ; \mathrm{K}_{3}=200 \mathrm{~kg} \mathrm{~K}{ }_{2} \mathrm{O} / \mathrm{ha}\right)$. dds = días después de la siembra. 
En los otros tratamientos la tendencia fue similar, con incrementos poco significativos del AF entre los 60 y 80 dds (Figura 1).

La mayor pérdida de AF se registró con 50.000 plantas $/ \mathrm{ha}^{-1}$ con todas las dosis de $\mathrm{K}$, aunque con 41.667 y 33.333 plantas/ha se notaron un incremento significativo en AF con 300 y 250 $\mathrm{kg} \mathrm{K}_{2} \mathrm{O}$ ha entre los 60 y 80 dds (Figura 1). Según Fernandes (2011), en cultivo de papa el K fue absorbido en mayor cantidad entre 41 y 61 dds. El $\mathrm{K}$ es el principal elemento responsable de la movilización de almidón desde las hojas al tubérculo (Nava et al., 2007).

En la figura 2 el IAF correspondió a un modelo polinómico de tercer grado con ajuste $\left(\mathrm{R}^{2}\right)$ superior a 0,61 con 50.000 y 41.667 plantas/ ha, la primera con 300 y $200 \mathrm{~kg} \mathrm{~K}$ O/ha y la segunda con $200 \mathrm{~kg} \mathrm{~K} \mathrm{O} / \mathrm{ha}$; el resto de los tratamientos se ajustaron al modelo de Hoerl con $\mathrm{R}^{2}$ superiores a 0,81 ; excepto, con 33.333 plantas/ha y $300 \mathrm{~kg} \mathrm{~K}$ O/ha que se ajustaron a un modelo cuadrático con un $\mathrm{R}^{2}$ de 0,85 ; con 50.000 plantas/ha y $250 \mathrm{~kg} \mathrm{~K} \mathrm{O} / \mathrm{ha}$ que se ajustaron a un modelo cuadrático recíproco con un $\mathrm{R}^{2}$ de 0,$88 ; 31.250$ plantas/ha y $250 \mathrm{~kg}$ $\mathrm{K}_{2} \mathrm{O} /$ ha se ajustaron a un modelo racional con un $\mathrm{R}^{2}$ de 0,8 .

El IAF con 41.667 (testigo) y 31.250 plantas/ha con $250 \mathrm{~kg} \mathrm{~K} \mathrm{~K}_{2} \mathrm{O} / \mathrm{ha}$, aumentaron el IAF entre los 60 y 80 dds, con un valor próximo a 2,0, luego la tendencia fue a disminuir hasta el final del ciclo (Figura 2). Algunos autores afirman que independientemente del sistema de producción utilizado para producir papa, casi siempre es necesario aplicar K (Nava et al., 2007; Singh y Lal, 2012). Una disminución de K puede ocasionar disminución de la materia seca MS y el AF (Santos, 2010).

Santos (2010) en el Centro de Colombia, en la variedad Guaneña reportó valores de IAF superiores a 6,0; sin embargo, el mismo autor reportavaloresentre2,5 y 4,9 parapapasembrada
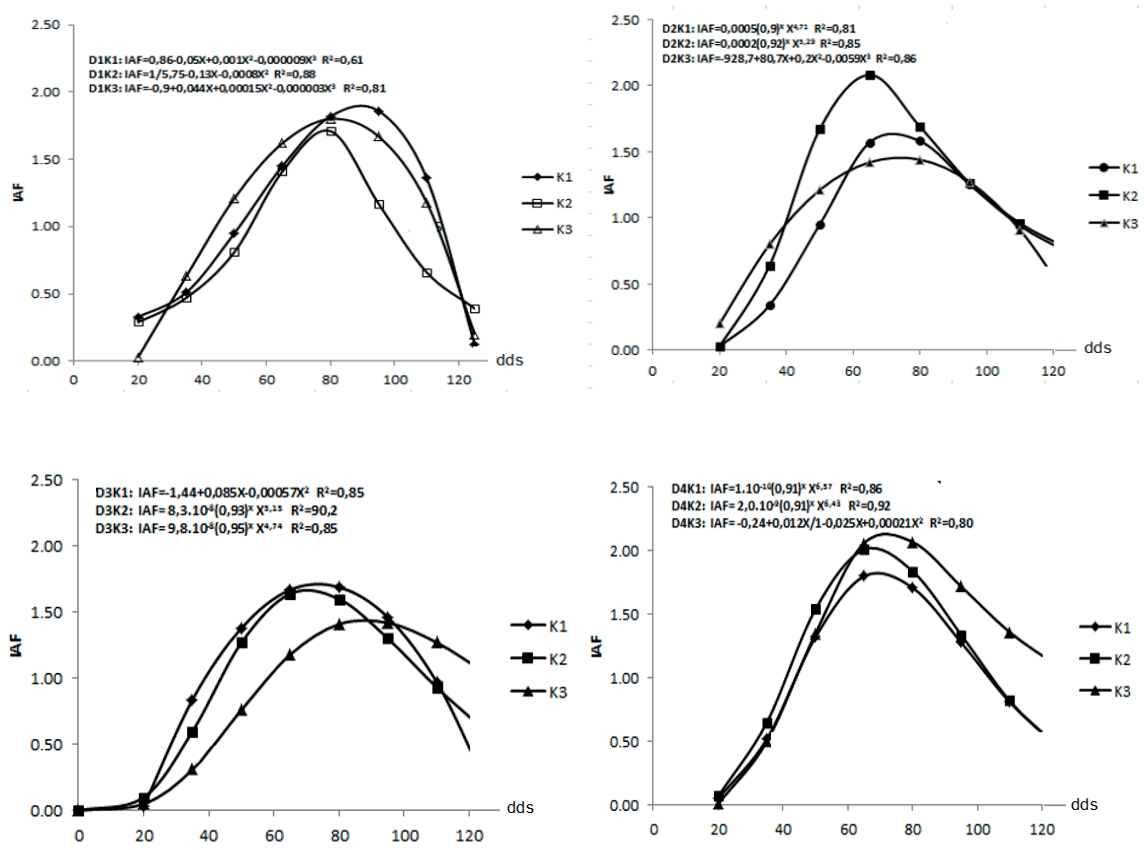

Figura 2. Modelos de regresión de mejor ajuste para índice de área foliar IAF de plantas de papa Solanum tuberosum var. Criolla Guaneña, en cuatro densidades de siembra y tres dosis de fertilizante potásico. $\left(\mathrm{D}_{1}=50.000\right.$ plantas $/ \mathrm{ha} ; \mathrm{D}_{2}=41.667$ plantas $/ \mathrm{ha} ; \mathrm{D}_{3}=33.333$ plantas $/ \mathrm{ha} ; \mathrm{D}_{4}=31.250$ plantas $/ \mathrm{ha} ; \mathrm{K}_{1}=300 \mathrm{~kg} \mathrm{~K} \mathrm{O}_{2}$ ha; $\left.\mathrm{K}_{2}=250 \mathrm{~kg} \mathrm{~K}{ }_{2} \mathrm{O} / \mathrm{ha} ; \mathrm{K}_{3}=200 \mathrm{~kg} \mathrm{~K} \mathrm{O} / \mathrm{ha}\right)$. dds = días después de la siembra. 
a alturas de $2.859 \mathrm{msnm}$, similar a la del estudio. En la figura 3 se observa en todos los tratamientos, que el ICC aumenta entre los 90 y 100 dds, que corresponde a la época de mayor acumulación de MS, donde se requiere una mayor demanda de fotoasimilados, debido al llenado de tubérculos (Santos et al., 2010), y luego decreció hasta el final del ciclo después de los 120 dds.

Con 50.000 plantas ha-1 y $250 \mathrm{~kg} \mathrm{~K}_{2} \mathrm{O} / \mathrm{ha}$ entre los 90 y 100 dds, se alcanzó un ICC de 40 g $\mathrm{m}^{-2}$ día $^{-2}$, que comparado con la dosis más baja de $\mathrm{K}$ fue mayor (Figura 3). Para sustentar una mayor cantidad de plantas durante el Ilenado de tubérculos, la dosis de $\mathrm{K}$ debe aumentar (Fernandes et al., 2011). Pero también el ICC fue mayor con 31.250 plantas/ha y $200 \mathrm{~kg} \mathrm{~K}_{2} \mathrm{O} /$ ha, posiblemente, al existir menor cantidad de plantas, hay mayor cubrimiento de las calles y se logra una mayor interceptación de la RFA que influye en el ICC, por la acumulación
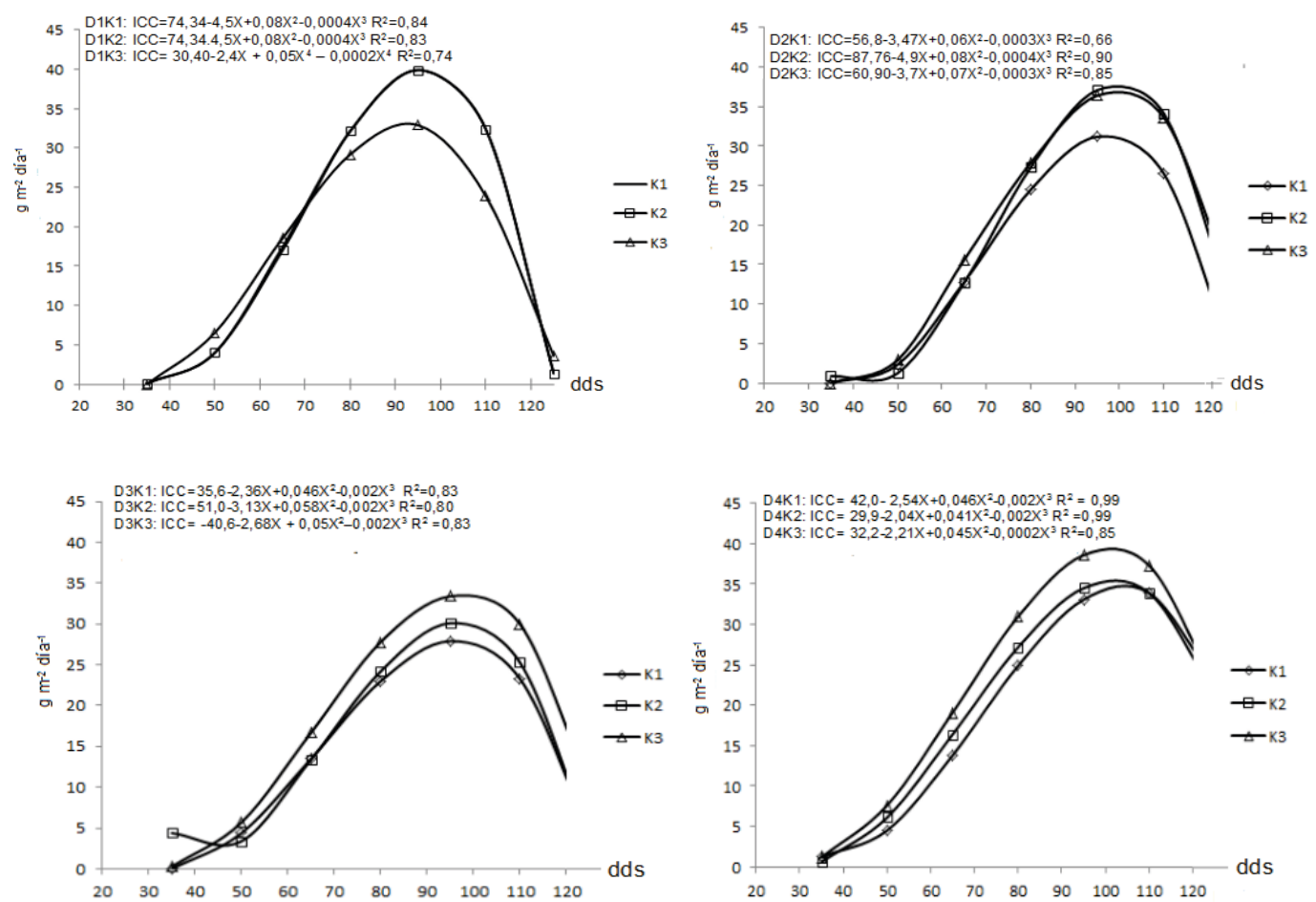

Figura 3. Comportamiento del índice de tasa de crecimiento del cultivo (ICC) de plantas de Solanum phureja var. Criolla Guaneña, en cuatro densidades de siembra y tres dosis de fertilizante potásico. $\left(D_{1}=50.000\right.$ plantas $/ \mathrm{ha} ; \mathrm{D}_{2}=41.667$ plantas $/ \mathrm{ha} ; \mathrm{D}_{3}=33.333$ plantas $/ \mathrm{ha} ; \mathrm{D}_{4}=31.250$ plantas $/ \mathrm{ha} ; \mathrm{K}_{1}=300 \mathrm{~kg} \mathrm{ha}^{-1} ; \mathrm{K}_{2}=250 \mathrm{~kg}$ $\left.\mathrm{ha}^{-1} ; \mathrm{K}_{3}=200 \mathrm{~kg} \mathrm{ha}^{-1}\right)$. dds = días después de la siembra. 
de 0,66. Mora-Aguilar et al. (2006) evaluaron cinco genotipos de Solanum tuberosum L. encontrandounaaltarelaciónentrelaprecocidad del genotipo y su eficiencia; los genotipos precoces como Alpha, Norteña y C-771A11 alcanzaron un IC entre 0,84 y 0,91 , mientras que en los tardíos el IC osciló entre 0,71 y 0,74.

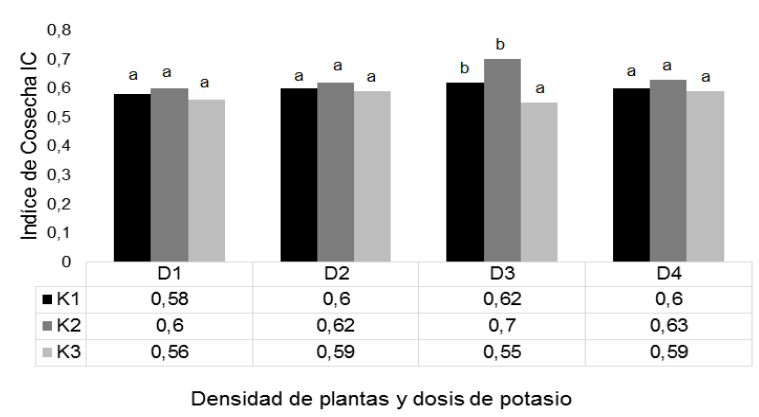

Figura 4. Valores de IC de papa Solanum tuberosum var. Criolla Guaneña, obtenidos con diferentes densidades de siembra y dosis de $\mathrm{K}_{2} \mathrm{O} .\left(\mathrm{D}_{1}=50.000\right.$ plantas/ha; $\mathrm{D}_{2}=41.667$ plantas/ha; $\mathrm{D}_{3}=33.333$ plantas/ha; $\mathrm{D}_{4}=31.250$ plantas $/ \mathrm{ha} ; \mathrm{K}_{1}=300 \mathrm{~kg} \mathrm{ha}^{-1}$; $\left.\mathrm{K}_{2}=250 \mathrm{Kg} \mathrm{ha}^{-1} ; \mathrm{K}_{3}=200 \mathrm{~kg} \mathrm{ha}^{-1}\right)$. Letras diferentes indican diferencias a una $\mathrm{P}<0,05$ según la prueba de Tukey.

En la figura 4, la tendencia a incrementos en el IC con 33.333 plantas/ha y las dosis más altas de $\mathrm{K}$, coincidieron con la baja tendencia a incrementos en las otras variables (Figuras 1, 2 y 3), lo cual pudo deberse a la influencia directa que ejercieron éstos dos factores de manejo de la producción en el aumento de la eficiencia del cultivo para la producción de fotoasimilados al final del ciclo (tuberización). Según Aguilar (2006), el rendimiento de tubérculos (IC) en el cultivo de papa está asociado a una mayor estabilidad fisiológica del cultivo en las fases tardías del desarrollo.

Los IC en la densidad de 33.333 plantas/ha, se mostraron influenciados por las dosis de K, siendo mayor con $250 \mathrm{~kg} \mathrm{ha}^{-1}$, y diferente a 200 $\mathrm{kg} \mathrm{ha}^{-1}(\mathrm{P}<0,05)$, con un IC menor y similar a $300 \mathrm{~kg} \mathrm{ha}^{-1}(\mathrm{P}>0,05)$ (Figura 4). La necesidad de fertilización de $K$ en papa, se explica por los altos requerimientos de la nutrición de la papa (lerna et al., 2011; Sifuentes et al., 2013).
Según Ñústez et al. (2009) al optimizar algunos factores de producción como el número de plantas y la fertilización, la eficiencia y/o capacidad de los vertederos (tubérculos) es mayor, y los rendimientos aumentan (Iwama, 2008; Streck, 2014).

Fernandes et al. (2011) demostraron que el K es fundamental en el desarrollo de los tubérculos, pero también mejora el desarrollo de los cultivos (Navas et al., 2007); ya que aumenta la resistencia a plagas y enfermedades del suelo y puede aumentar la tolerancia a condiciones climáticas adversas (heladas, granizadas, otros) (Mohr et al., 2012).

La mayor demanda de asimilados ocurre en las etapas iniciales en hojas y tallos, pero al iniciarse el desarrollo de los tubérculos, éstos se constituyen en el principal órgano de almacenamiento (Criollo y García, 2009), donde el $\mathrm{K}$ juega un papel importante.

\section{CONCLUSIONES}

Las densidades de siembra y dosis de K evaluadas influyeron en el comportamiento de las variables fisiológicas de AF, IAF e ICC de papa Solanum tuberosum Grupo Phureja var. Criolla Guaneña, expresando diferencias en el ajuste al modelo matemático.

Con 31.250 plantas/ha y $250 \mathrm{~kg} \mathrm{~K} \mathrm{O} / \mathrm{ha}$ se alcanzan incrementos en el AF e IAF entre los 60 y 80 dds y con $200 \mathrm{~kg} \mathrm{~K}$ O/ha en el ICC entre los 90 y 100 dds de papa Solanum tuberosum Grupo Phureja var. Criolla Guaneña.

El testigo (41.667 plantas/ha y $250 \mathrm{~kg} \mathrm{~K} \mathrm{O} / \mathrm{ha}$ ) se asocia con un mayor ICC entre los 90 y 100 dds, pero no con el IC.

EI $\mathrm{K}$ influye en el IC, ya que incrementó con las dosis más altas (250 y 300 kg K2 O/ha), a diferencia de la dosis más baja $(200 \mathrm{~kg} \mathrm{~K} \mathrm{O} /$ ha) y una densidad de 33.333 plantas/ha de 
Solanum tuberosum Grupo Phureja var. Criolla Guaneña bajo las condiciones del Altiplano de Pasto.

\section{REFERENCIAS}

Abramoff, M., Magalhães, P. y Ram, S. 2004. Image processing with ImageJ, IgiturArchief - Utrecht Publishing and Archiving Service. Internet, http://www. temoa.info/es/node/321343 [8 junio 2012].

Aguilar, M. 2006. Análisis de crecimiento y de relaciones fuente - demanda en dos variedades de papa (Solanum tuberosum L.). Revista Fitotecnia Mexicana. 29(2):145-158.

Becerra, L., Navia, S. y Ñustez, C. 2007. Efecto de niveles de fosforo y potasio sobre el rendimiento del cultivar "Criolla Guaneña" en el departamento de Nariño. Revista de la Asociación Latinoamericana de la Papa. 14(1):51-60.

Criollo, H. y García, J. 2009. Effect of planting density on the growth of radish (Raphanus sativa L.) plants under Greenhouse conditions. Revista Colombiana de Ciencias Hortícolas. 3 (2):210-222. https:// doi.org/10.17584/rcch.2009v3i2.1214

De La Casa, A., Ovando, G., Bressanini, L., Martínez, J., Ibarra, E. y Rodríguez, Á. 2008. El índice de área foliar en papa estimado a partir de la cobertura del follaje Leaf area index in potato estimate from canopy cover. Agronomía Tropical. 58(1): 61-64.

Fernandes, A., Soratto, R. e Silva, B. 2011. Extração e exportação de nutrientes em cultivares de batata: I - macronutrientes. Revista Brasileira de Ciência do Solo. 35(6):20392056. https://doi.org/10.1590/S010006832011000600020

Garnier, E., Shipley, B., Roumet, C and Laurent, G. 2004. A standardized protocol for the determination of specific leaf area and leaf dry matter content. Functional
Ecology. 15(5): 688-695. https://doi. org/10.1046/j.0269-8463.2001.00563.x

Holdridge, I. 1990. Determination of World Plant Formations from Simple Climatic Data. Science. 105(2727):367-368.

lerna, A., Pandino, G., Lombardo, S., Mauromicale, G. 2011. Tuber yield, water and fertilizer productivity in early potato as affected by a combination of irrigation and fertilization. Agricultural Water Management. 101:35-41. https:// doi.org/10.1016/j.agwat.2011.08.024

Instituto De Hidrología, Meteorología y Estudios Ambientales (IDEAM). 2013. Datos meteorológicos. Estación meteorológica de Obonuco. Pasto.

Iwama, K. 2008. Physiology of the Potato: New Insights into Root System and Repercussions for Crop Management. Potato Research. 51(3-4):333-353. https:// doi.org/10.1007/s11540-008-9120-3

Madroñero, I., Rosero, J., Rodríguez, I., Navia, J., y Benavides, C. 2013. Caracterización morfoagronómica de genotipos promisorios de papa criolla (Solanum tuberosum L. Grupo Andigenum) en Nariño. Temas Agrarios. 18(2):50-66. https://doi.org/10.21897/rta.v18i2.716

Mahmud, A., Hoque, M., Mamun, A., Bazzaz, M. and Hossain, M. 2012. Yield and profitability of potato as influenced by intra-row spacing and number of eye in cut tuber in northern region of Bangladesh. Annals of Bangladesh Agriculture. 14:111-124.

Mohr, R. and Tomasiewicz, D.L. 2012. Effect of rate and timing of potassium chloride application on the yield and quality of potato (Solanum tuberosum L. 'Russet Burbank'). Canadian Journal of Plant Science. 92:783-794. https://doi. org/10.4141/cjps2011-195

Mora-Aguilar, R., Ortiz-Cereceres, J., RiveraPeña, A., Mendoza-Castillo, M., Colinas- 
León, M. y Lozoya-Saldaña, H. 2006. Índices de eficiencia de genotipos de papa establecidos en condiciones de secano. Revista Chapingo Serie Horticultura. 12(1): 85-94. https://doi.org/10.5154/r. rchsh.2004.09.049

Nava, G., Dechen, A. e Luchi, V. 2007. Produção de tubérculos de batatasemente em função das adubações nitrogenada, fosfatada e potássica. Horticultura Brasileira. 25:365370. https://doi.org/10.1590/S010205362007000300009

Ñústez, C., Santos, M. y Segura, M. 2009. Acumulación y distribución de materia seca de cuatro variedades de papa (Solanum tuberosum L.) en Zipaquirá, Cundinamarca (Colombia). Revista Facultad Nacional de Agronomía Medellín. 62(1): 4823-4834.

Ríos, J. 2010. Determinación del Efecto de Diferentes Niveles de Fertilización en Papa (Solanum tuberosum ssp. Andigena) Diacol Capiro en un Suelo con Propiedades ándicas de Santa Rosa de Osos, Colombia. Revista Facultad Nacional de Agronomía Medellín. 63(1): 5225-5237.

Rodríguez, I., Corchuelo, G. y Ñústez, C. 2004. Influencia del espaciamiento entre plantas sobre la morfología y el crecimiento de la papa (Solanum tuberosum L. Cv. Parda pastusa) bajo dos ambientes contrastantes. Agronomía Colombiana. 21(3): 210-219.

Rodríguez, L., Ñustez, C. y Estrada, N. 2009. Criolla Latina, Criolla Paisa y Criolla Colombia, nuevos cultivares de papa criolla para el departamento de Antioquia (Colombia). Agronomía Colombiana. 27(3):289-303.

Rojas, S. 2014. Productividad de diez cultivares promisorios de papa chaucha (Solanum tuberosum, grupo Phureja) de la región Cajamarca. Scientia Agropecuaria. 5: 165-175.
Santos, M. 2010. Evaluación del crecimiento, desarrollo y componentes de rendimiento de cuatro cultivares de papa criolla en dos localidades del departamento de Cundinamarca. Tesis Ingeniero Agrónomo, Universidad Nacional de Colombia, Bogotá.

Santos, M., Segura, M. y Ñústez, C. 2010. Análisis de Crecimiento y Relación Fuente-Demanda de Cuatro Variedades de Papa (Solanum tuberosum L.) en el Municipio de Zipaquirá (Cundinamarca, Colombia). Revista Facultad Nacional de Agronomía Medellín. 63(1):5253-5266.

Schneider, C. 2012. "NIH Image para ImageJ: 25 años de análisis de imagen". Nature Methods. 9: 671-675. https://doi. org/10.1038/nmeth.2089

Sifuentes, I., Ojeda, E., Mendoza, P., Macías, C., Rúelas, J., Del Rosario, J. e Inzunza, M. 2013. Nutrición del cultivo de papa (Solanum tuberosum L.) considerando variabilidad climática en el "Valle del Fuerte", Sinaloa, México. Revista mexicana de ciencias agrícolas. 4(4):585597.

Singh, S.K., and Lal, S. 2012. Effect of Potassium Nutrition on Potato Yield, Quality and Nutrient Use Efficiency under Varied Levels of Nitrogen Application. Potato Journal. 39(2):155-165.

Streck, N., Garrido, D., Junior, A., Fernandes, L., Schmitz, T., Trevisan, A. and Rocha, M. 2014. Effect of plant spacing on growth, development and yield of cassava in subtropical environment. Bragantia. 73(4):407-415. https://doi. org/10.1590/1678-4499.0159.

Unigarro, A. y Carreño, M.R. 2005. Métodos químicos para el análisis de suelos. San Juan de Pasto: Colombia. 72p. 